\title{
Expected Changes of SNe with Redshift due to Evolution of Their Progenitors
}

\author{
Inma Domínguez, ${ }^{1}$ Peter Höflich, ${ }^{2}$ Oscar Straniero, ${ }^{3}$ Marco Limongi ${ }^{4}$ and \\ Alessandro Chieffi ${ }^{5}$ \\ 1 Universidad de Granada, Granada, Spain \\ inma@ugr.es \\ 2 Universidad de Texas, Austin, USA \\ 3 INAF-Osservatorio Astronomico di Collurania, Teramo, Italy \\ 4 INAF-Osservatorio Astronomico di Roma, Monteporzio, Italy \\ 5 Istituto di Astrofisica Spaziale, CNR, Roma, Italy
}

Summary. We have analyzed the influence of the stellar populations, from which SN progenitors come, on the observational outcome, including the metal free Pop. III. We use our models to study the evolution of the progenitor, the subsequent explosion and the light curves. For Type Ia, the variation of the main sequence mass of the progenitor of the exploding WD produces an offset in the maximum-decline relation of $0.2 \mathrm{mag}$. This effect is critical for the use of high redshift Type Ia SNe as cosmological standard candles. In contrast, the metallicity does not change the above relation (at maximum, $\Delta M_{V} \leq 0.06 \mathrm{mag}$ ). For Type II, we find a dependence of the light curve properties with both main sequence mass and metallicity of the progenitor, and we identify a rather homogeneous subclass, "Extreme II-P," that may be used as a quasi-standard candle. Note that, although not as good as Type Ia for distance determinations, Type II are expected to have occurred since the first stars were formed.

\section{Introduction}

Due to their high brightness, SNe are among the best candidates to measure distances on cosmological scales; in particular, Type Ia SNe (SNe Ia) are the favorites as they are brighter by about 1 to 2 magnitudes than other class of SNe and show very homogeneous properties. Recently, SNe Ia observed at high redshifts, $\mathrm{z}$, (see $[31,35]$ ) have provided results that are consistent with a Universe composed mainly of "dark energy" or, equivalent, with a positive cosmological constant, $\Omega_{\Lambda} \sim 0.7$. Note that this conclusion relies on the application of an empirical relation between light curve (LC) shape and maximum luminosity which is obtained for the well observed nearby SNe Ia occurring in galaxies at known distance [31, 34].

To understand the nature of this dark energy, we need to observe standardcandles at high $z$. The quantity and quality of the data is increasing due to systematic searches, space telescopes and 8m-class ground telescopes. However, SNe or other standard-candles are calibrated locally, and their application to very high z may be limited by evolutionary effects. In fact, there is 
observational evidence indicating a correlation of SN properties with those of their host galaxies $[2,3,11,22,38]$.

$\mathrm{SNe}$ Ia are thought to be the thermonuclear explosion of $\mathrm{CO}$ white-dwarfs [20] triggered by mass accretion from a companion. The proposed scenarios are merging of WDs by gravitational radiation (double degenerate) [21, 39], and accretion of $\mathrm{H}$ and/or He from a non-degenerate companion (single degenerate) [40]. Moreover, they occur in elliptical galaxies indicating that some of their progenitors, at least, have evolutionary time-scales comparable to the Hubble time and, thus, at high z SNe Ia should be rare.

The other class of SNe, core collapse $\mathrm{SNe}$, are thought to be the explosion of massive stars (greater than $\sim 10 M_{\odot}$ ) caused by the collapse of its central parts into a neutron star or a black hole. Their evolutionary time scales are short compared to the age of the universe even at high $\mathrm{z}[26,37,42]$. Core collapse $\mathrm{SNe}$ show a wide range of brightness, up to 6 magnitudes, and properties of their light curves $[10,30,43]$ which prevents their use as standard-candles. However, our knowledge of the event is improving and it may be possible to derive the absolute magnitude in a similar way as Type Ia if appropriate empirical correlations can be identified (see Hamuy, this volume and [12]). These objects will occur soon after the initial star formation period and, therefore, can be used to probe the structure of the universe at high $\mathrm{z}$ (e.g., at $z \sim 5$ ). At that epoch, galaxies are expected to be small and dim and core collapse supernovae may be the brightest objects in the Universe [27].

It is expected that, going back in time, the stellar population of the SN progenitors would be composed of more low metallicity and rapidly evolving, more massive, stars. In this work we focus, mainly, on the exploration of the sensitivity of the light curve characteristics on the underlying progenitor properties: initial mass and metallicity $(\mathrm{Z})$.

\section{Numerical Models}

\subsection{Stellar Evolution}

The evolution of selected models in the mass range from 1.5 to $25 \mathrm{M}_{\odot}$ and $\mathrm{Z}$ between 0 (Pop.III) and 0.02 (solar) have been computed from the pre-main sequence to the TP-AGB phase in the case of the low and intermediate mass stars and to the onset of core collapse for the massive stars. We have not included rotation neither mass loss.

All models have been computed by means of the evolutionary code FRANEC (rev 4.2) and are extensively described in [7, 9, 25]. The details of the FRANEC has been presented in $[4,5,36]$. 


\subsection{Explosions and Light Curves}

Based on the previous models, the explosion, detailed post-processing and light curves are computed by means of a $1 \mathrm{D}$ radiation-hydrodynamic code (see $[7,9,16]$ ). All parameters are fixed in order to analyze the sensitivity of the observed properties to the initial mass and metallicity of the progenitors.

For Type Ia we have considered delayed detonation explosions because models based on this explosion mechanism reproduce monochromatic light curves and spectra reasonable well, including the maximum-LC shape relation (see for example $[14,15,24,29,41]$. The description of the velocity of the deflagration front is based on 3D simulations [8, 23, 28]; model parameters, transition density and ignition density, are fixed and have been chosen to reproduce a typical SN Ia.

For Type II, the explosion is triggered artificially by depositing energy above the mass cut, fulfilling the requirement that the final kinetic energy and the ${ }^{56} \mathrm{Ni}$ mass is the same in all models.

\section{Results}

\subsection{Type Ia}

We have connected the initial mass and metallicity of the WD progenitor to the light curves and spectral properties of SNe Ia. All the potential progenitors of the WD have been studied. The key parameter is the integrated $\mathrm{C} / \mathrm{O}$ ratio, the fuel, within the Chandrasekhar mass WD. In general, changes are small because the nuclear energy released by a complete burning (into iron group ashes) of a pure Carbon WD is about $10 \%$ larger than that released by a pure Oxygen WD with the same mass.

We find that the initial mass of the progenitor modifies the average $\mathrm{C} / \mathrm{O}$ in the WD up to a $22 \%$ and, as a consequence, the ${ }^{56} \mathrm{Ni}$ mass produced by the explosion and the kinetic energy (for details see [9] and also [17]). Progenitors with greater masses (smaller $\mathrm{C} / \mathrm{O}$ rate within the WD and consequently less amount of ${ }^{56} \mathrm{Ni}$ ) produce less luminous and slightly faster decline LCs. In particular, the initial mass alters the maximum-LC shape relation, which may be offset by up to $0.2 \mathrm{mag}$. Notice that for a detailed analysis of the nature of the dark energy an accuracy of $0.05 \mathrm{mag}$ is required [1].

In addition, for these massive progenitors, kinetic energies and expansion velocities are also smaller, down to $2000 \mathrm{~km} / \mathrm{s}$. This correlation between LC shape and expansion velocity may be used to reduce the scatter in the empirical relation from which the maximum luminosity is obtained.

The dependence of the total $\mathrm{C} / \mathrm{O}$ on the initial mass is mainly due to the different extension of the convective core during the central He burning phase. In fact, the internal structure of an exploding WD may be schematically divided in two distinct regions: an internal one, where $\mathrm{C}$ is significantly 
depleted (about $25 \% \mathrm{C}$ and $75 \% \mathrm{O}$ ) and an external one, where $\mathrm{C} / \mathrm{O}$ is about 1. The internal region is built in during the core He-burning phase, while the external one is left by the shell burning (AGB or accretion phase). Since the extension of the central region (C depleted) coincides with the convective core of the He-burning progenitor, and since more massive progenitors have a larger convective cores, the larger is the progenitor mass, the smaller is the final (pre-explosive) $\mathrm{C} / \mathrm{O}$.

In contrast, the dependence of the size of this inner C-depleted region with metallicity is much weaker and not monotonic. In fact, the metallicity of the progenitor does not influence the average $\mathrm{C} / \mathrm{O}$ ratio within the $\mathrm{WD}$; changes are smaller than $9 \%$ and, as a consequence, the amount of ${ }^{56} \mathrm{Ni}$ produced in the explosion and the kinetic energies are rather similar (however, see also [18]). For this reason, metallicity does not influence the maximum luminosity $\left(\Delta M_{V} \leq 0.06 \mathrm{mag}\right)$, nor the LC shape.

Finally, we want to stress, that the final integrated $\mathrm{C} / \mathrm{O}$ ratio within the pre-explosive Chandrasekhar mass WD depends on the scenario; mergers (double degenerate) are expected to have a smaller $\mathrm{C} / \mathrm{O}$ ratio compared to single degenerates. The reason is that, in the case of merging, two central carbon depleted region corresponding to the two WDs, are added.

\subsection{Type II}

First, we want to mention the limitation of our theoretical study; we have explored a very limited parameter space (see Hamuy, these Proceedings, and [13]) and to compare with observations, different ${ }^{56} \mathrm{Ni}$ masses and kinetic energies should be considered and mass loss included during the evolution of the progenitor.

We find (see $[7,19]$ for details) that all the Pop. III models end up as Blue Super Giants (BSG) while all the solar metallicity ones end up as Red Super Giants (RSG). At intermediate Z, the more massive stars end up as BSG while the less massive ones end up as RSG. The limiting mass depends on $\mathrm{Z}$. In general, the stellar radius depends on the opacity of the envelope; the lower the metallicity, the lower the opacity and, as a consequence, the structure is more compact.

If the progenitor is a RSG, the brightness during the plateau phase, which lasts more than 50 days, is nearly constant, $M_{V} \sim-17.5$, and quite insensitive to changes of the initial mass $\left(\Delta M_{V} \leq 0.07 \mathrm{mag}\right)$. Increasing the kinetic energy by a factor of 2 , results in a slightly brighter plateau, $\Delta M_{V} \leq 0.3 \mathrm{mag}$. This makes this sub-class, which we call "Extreme II-P," a quasi-standard candle.

The similarity of the LCs is caused by the similarity of the density structures of the RSG envelopes, which show flat density gradients at the photosphere, which during the plateau phase is located at the H-recombination front. A self-regulating mechanism is at work: an increase in the energy release causes a heating of the photosphere and a reduction of the energy production 
rate and vice versa. Because the density slope of the photosphere is flat and slowly changing, the luminosity profile of the light curves is rather flat.

Moreover, the unique light curves of Extreme II-P allow photometric identification and so, permit their observation from ground 8m-class telescopes up to a redshift of $\mathrm{z} \sim 3$. Space telescopes, like SIRTF, would push this limit even further and the NGST is expected to detect SNII up to $z \sim 10$.

BSG progenitors produce sub-luminous events, 1.5 magnitudes fainter than SNII which come from RSGs. The light curve presents, instead of the plateau, a long-lasting phase of increasing brightness. In this case the selfregulating mechanism does not work due to the steep density gradients.

Finally, these studies are very preliminary, key problems, concerning the identification of the progenitors (Type Ia) and the explosion mechanisms (Type Ia and Type II), are still to be solved, and, besides, we do not know how the stellar populations evolve with redshift.

\section{References}

1. A. Albrecht, J. Weller: Astron. Astrophys. Suppl. 197, 6106 (2000)

2. D. Branch, W. Romanishing, E. Baron: Astrophys. J. 465, 73 (1996)

3. E. Cappellaro et al. : Astron. Astrophys. 322, 421 (1997)

4. A. Chieffi, O. Straniero: Astrophys. J. Suppl. 71, 48 (1989)

5. A. Chieffi, M. Limongi, O. Straniero: Astrophys. J. 502, 737 (1998)

6. A. Chieffi, M. Limongi: Astrophys. J. 577, 281 (2002)

7. A. Chieff, I. Domínguez, P. Höflich, M. Limongi, O. Straniero: Mon. Not. R. Astron. Soc. 345, 111 (2003)

8. I. Domínguez, P. Höflich: Astrophys. J. 528, 854 (2000)

9. I. Domínguez, P. Höflich, O. Straniero: Astrophys. J. 557, 279 (2001)

10. A.V. Filippenko: In: Cosmic Explosions. eds. S. Holt, W.W. Zhang (AIP: New York, 2000)

11. M. Hamuy et al. : Astron. J. 120, 1479 (2000)

12. M. Hamuy, P.A. Pinto: Astrophys. J. Lett. 566, L63 (2002)

13. M. Hamuy: Astrophys. J. 582, 905 (2003)

14. P. Höflich: Astrophys. J. 443, 89 (1995)

15. P. Höflich, A. Khokhlov, J.C. Wheeler: Astrophys. J. 444, 831 (1995)

16. P. Höflich, A. Khokhlov: Astrophys. J. 457, 500 (1996)

17. P. Höflich, J.C. Wheeler, F.K. Thielemann: Astrophys. J. 495, 617 (1998)

18. P. Höflich, K. Nomoto, H. Umeda, J.C. Wheeler: Astrophys. J. 528, 590 (2000)

19. P. Höflich, O. Straniero, M. Limongi, I. Domínguez, A. Chieffi: Rev. Mex. Astron. Astrofis. Conf. Series 10, 157 (2001)

20. P. Hoyle, W.A. Fowler: Astrophys. J. 132, 565 (1960)

21. I.J. Iben, A.V. Tutukov: Astrophys. J. Suppl. 54, 335 (1984)

22. V.D. Ivanov, M. Hamuy, P.A. Pinto: Astrophys. J. 542, 588 (2000)

23. A. Khokhlov: Astrophys. J. 457, 695 (1995)

24. E.J. Lentz, E. Baron, D. Branch, P. Hauschildt, P.E. Nugent: Astrophys. J. 530, $966(2000)$

25. M. Limongi A. Chieffi A., O. Straniero: Astrophys. J. Suppl. 129, 625L (2000) 
26. J. Maza S. van den Bergh: Astrophys. J. 204, 519 (1976)

27. J. Miralda-Escudé, M.J. Rees: Astrophys. J. 478, 57 (1997)

28. J.C. Niemeyer, W. Hillebrandt: Astrophys. J. 452, 779 (1995)

29. P.E. Nugent, E. Baron, P. Hauschildt, D. Branch: Astrophys. J. 485, 812 (1997)

30. F. Patat, R. Barbon, E. Cappellaro, M. Turatto: Astron. Astrophys. 282, 731 (1994)

31. S. Perlmutter et al. : Astrophys. J. 517, 565 (1999)

32. M.M. Phillips et al. : Pub. Astron. Soc. Pacific 90, 592 (1987)

33. M.M. Phillips P. Lira, N.B. Suntzeff, R.A. Schommer, M. Hamuy, J. Maza: Astron. J. 118, 1766 (1999)

34. A.G. Riess, W.H. Press, R.P. Kirshner: Astrophys. J. 473, 588 (1996)

35. B.P. Schmidt et al. : Astrophys. J. 507, 46 (1998)

36. O. Straniero, A. Chieffi, M. Limongi: Astrophys. J. 490, 425 (1997)

37. G. Tammann: In: NATO-ASI on Supernovae: A Survey of Current Research. eds. M.J. Rees, R.J. Stoneham (Reidel: Dordrecht, 1982) p. 371

38. L. Wang, P. Höflich, J.C. Wheeler: Astrophys. J. Lett. 487, L29 (1997)

39. R.F. Webbink: Astrophys. J. 277, 355 (1984)

40. J. Whelan, I. Iben, Jr.: Astrophys. J. 186, 1007 (1973)

41. J.C. Wheeler, P. Höflich, R. Harkness, J. Spyromilio: Astrophys. J. 496, 908 (1998)

42. S.E. Woosley, T.A. Weaver: Ann. Rev. Astron. Astrophys. 24, 205 (1986)

43. T.R. Young, D. Branch: Astrophys. J. Lett. 342, L79 (1989) 